\title{
NEW WINDOW DESIGN OPTIONS FOR CEBAF ENERGY UPGRADE
}

DOE $\begin{aligned} & E R \text { to } 150 \\ & \text { L. Phillips, J. Mammosser, and V. Nguyen, Thomas Jefferson National Accelerator Facility, }\end{aligned}$ 12000 Jefferson Avenue, Newport News, VA 23606 USA

CONFtract $-970503-165$

As the Jefferson Laboratory upgrades the existing CEBAF electron accelerator to operate at higher energies, the fundamental power coupler windows will be required to operate with lower $\mathbf{R F}$ dissipation and increased immunity to radiation from cavity field emission. New designs and modifications to existing designs which can achieve these goals are described.

\section{INTRODUCTION}

The Continuous Electron Beam Accelerator Facility (CEBAF) located at Thomas Jefferson National Accelerator Facility, in Newport News, Virginia is fully operational. The accelerator consists of 165 hermetically sealed cavity pairs, each providing $5 \mathrm{MeV}$ energy gain per pass for the machine. RF power from a $5 \mathrm{KW}$ klystron, coupled through two RF windows, drives each cavity independently to accelerating gradients in excess of $5 \mathrm{MV} / \mathrm{m}$. One of these windows is made of ceramic and, attached to the cavity, operates at $2 \mathrm{~K}$ (fig 1). The history and performance of this cold window, a discussion of needs for new designs for the machine energy upgrade and the concepts being developed at Jefferson Lab will be discussed.

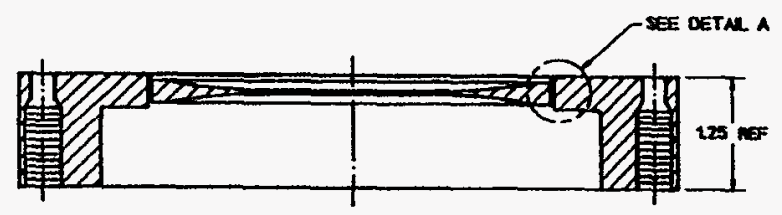

Figure 1

\section{ORIGINAL DESIGN}

The original input coupler for the CEBAF accelerator includes two windows in series (Fig. 2). This design attempts to minimize the contamination of the cavity pairs by hermetically sealing the cavity pair early in the assembly process. A ceramic window, indium seals and gate valves at the ends of the pair provide the hermiticity. The design requirements for the cold window were: low voltage standing wave ratio (VSWR) at the fundamental frequency, propagation of the certain higher order modes (HOM) which are not adequately damped by the HOM absorbers, and a cryogenically compatible metal-ceramic design with low particulate generation. The window design (Fig. 1) consists of a thin ceramic with small compensating irises to minimize VSWR both at the fundamental and HOM frequencies. The resulting VSWR is 1.1 at $1500 \mathrm{MHz}$ and 1.3 at $1800 \mathrm{MHz}$.

DISTRIBUTION OF THIS DOCUMENT IS UNLIMITED

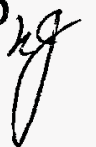

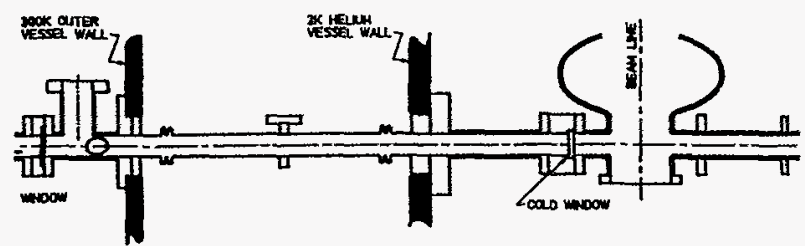

Figure 2

\section{PERFORMANCE OF CEBAF WINDOW}

The most serious performance limitation experienced with the CEBAF cold window is its contribution to machine interlock trips $[1,2,3]$. The fundamental power coupler, consisting of a cold window separated from a warm window by an evacuated waveguide, is protected by two primary interlocks. One interlock is a photomultiplier tube viewing the waveguide region between windows that shuts off RF klystron power if light appears. A second interlock shuts off RF power if the pressure in the waveguide rises above $1 \times 10^{-7}$ Torr. These interlocks protect both windows from damage due to sustained RF discharge. There are two types of coupler related interlock trips which are not distinguishable in operation as to cause.

A pressure burst in the waveguide vacuum space can occur from an RF transient or phase glitch which rapidly alters the thermal profile of the waveguide releasing hydrogen, for example, from the cold end of the waveguide where the thermal time constant is short. Such transients are known to occur when RF power is switched off to an entire cryomodule at once, tripping all cavities in the cryomodule. A pressure burst can cause a gas discharge producing both a light trip and a vacuum trip.

Surface flashover on the window will also produce a light trip and initiates gas desorption, producing a vacuum trip as well. This leaves some ambiguity with respect to the cause of a trip.

Cavities tripping at regular intervals however indicate that perhaps $90 \%$ of the coupler-related interlock trips in CEBAF are due to surface flashover on the ceramic [2] resulting from a charging process.

A number of studies have shown that the ceramic window becomes charged from electronic activity in the $\mathrm{RF}$ cavity in the presence of field emission. Ultimately, the surface charge builds to some threshold and is relieved by surface flashover. The RF fields alone at the window are not sufficient to induce surface flashover in the absence of surface charge. This charging effect followed by a discharge leads to a periodicity in the interlock trips for a given cavity operated with field emission at a fixed gradient. The period is observed to decrease with increasing RF power dissipated in field emission in a test cavity, consistent with a model of surface charging and brealown threshy

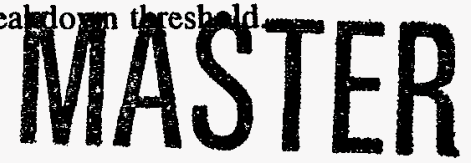




\section{DISCLAIMER}

This report was prepared as an account of work sponsored by an agency of the United States Government. Neither the United States Government nor any agency thereof, nor any of their employees, make any warranty, express or implied, or assumes any legal liability or responsibility for the accuracy, completeness, or usefulness of any information, apparatus, product, or process disclosed, or represents that its use would not infringe privately owned rights. Reference herein to any specific commercial product, process, or service by trade name, trademark, manufacturer, or otherwise does not necessarily constitute or imply its endorsement, recommendation, or favoring by the United States Government or any agency thereof. The views and opinions of authors expressed herein do not necessarily state or reflect those of the United States Government or any agency thereof. 


\section{DISCLAMMER}

Portions of this document may be illegible in electronic image products. Images are produced from the best available original document. 
For these reasons it is necessary to eliminate surface charging of the RF windows in order to take advantage of higher gradients with reduced interlock trip rate. Some smaller percentage of trips could be eliminated by improving the waveguide vacuum stability.

A second performance issue with the cold ceramic window is heat load and economics. The ceramic window loss at $5 \mathrm{MV} / \mathrm{m}$ is about 0.5 watts but at 10 $\mathrm{MV} / \mathrm{m}$ it would be 2.0 watts. At $2 \mathrm{~K}$ this represents an objectionably high power cost.

Sources of loss for this window were examined through a series of waveguide resonator tests. A window model with various interchangeable parts was tested at 2 $\mathrm{K}$ by measuring the $\boldsymbol{Q}$ of the resonator under differing test conditions. Different ceramic materials were compared as well as ceramic metallization and position of the window in the resonator standing wave pattern.

In general, very-high-purity ceramics such as sapphire and pure polycrystalline aluminas have lower loss than glass-bearing ceramics. Ceramic metallization was the dominant source of heat, and total loss was highest when the window was placed in an electric field maximum.

\subsection{Punctures}

Very small window punctures are also related to the presence of field emission in the cavity. These are very small leaks, invisible to the naked eye and induced by window charging/discharging [1]. Several such leaks are know to exist in the accelerator. Their impact on machine operation is difficult to assess since the primary purpose of the cold window is to reduce particulate contamination of the cavity during initial assembly and installation in the cryomodule. Once a vacuum is maintained on both sides of the window it continues to serve this function even with a puncture. The major risk might be the possibility of shedding small particulates of ceramic from the puncturing process which could end up in the cavity.

\section{LIMITATIONS OF CEBAF WINDOW FOR ENERGY UPGRADE}

The current machine energy upgrade plan requires each cavity to operate at an accelerating gradient around 10 $\mathrm{MV} / \mathrm{m}$ at $Q$-value of $5 \times 10^{9}$. With $60 \%$ of the cavities limited by field emission in vertical test at gradients of $7-8 \mathrm{MV} / \mathrm{m}$, the cavities for upgrade will most likely need to operate with some field emission, and this could produce an objectionable machine trip rate if modifications are not implemented. In order to reduce this trip rate, the window ceramic will have to be shielded from electron spray and radiation. Currently 153 cavities in the accelerator are gradient-limited by arcing [5]. At present, care has been taken in understanding each cavity trip limitation, and gradient settings are administratively controlled to minimize the impact on machine performance.

A second performance issue with the ceramic window is one of economics. The ceramic window adds to the power dissipated in the $2 \mathrm{~K}$ bath during operation and any reduction of this power could be a cost savings. The loss generated by the ceramic window has two components, the metallization of the ceramic which is the dominant loss and dielectric loss of the ceramic. Design for higher-energy operation must address this heat load.

Cold window punctures are a product of field emission in the cavity. There are certain cavities which have repeatedly punctured replacement windows. Figure 3 shows cavity IA174 RF performance in a vertical test that resulted in a punctured window. This was typical of the type of performance that resulted in a ceramic puncture, with heavy field emission at low accelerating gradient. In this particular case, the puncture was located in the radius where the ceramic is ground from $6 \mathrm{~mm}$ to $1 \mathrm{~mm}$ and $4.3 \mathrm{~cm}$ from the end. Punctures typically were located at this ground radius. The circumstances producing punctures are now sufficiently understood that we can expect this problem to disappear when surface charging of the window ceramic is eliminated.

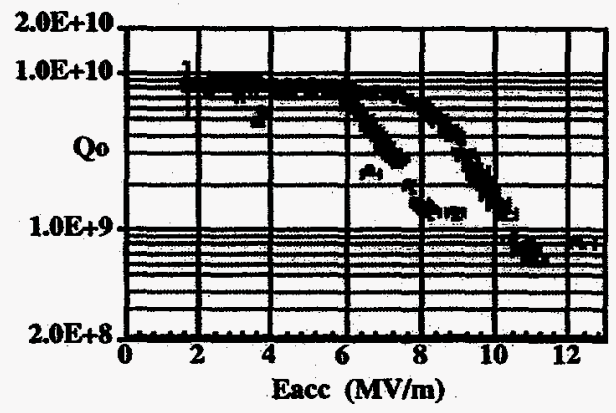

Figure 3 IA174 vertical test data that resulted in a ceramic puncture.

\section{DESIGN MODIFICATIONS FOR THE ENERGY UPGRADE}

\section{I $2 \mathrm{~K}$ Heat Load}

RF loss occurs primarily in the ceramic braze metallization, and its reduction could be accomplished several ways.

One is to reduce the total area of metallized ceramic exposed to RF current. Test windows have been constructed with braze areas reduced to one half of the original production value without sacrificing seal integrity. Using this technique window loss, as measured in a waveguide resonator, has been reduced to less than half the production value.

Another opportunity presently being explored is to shield the seal area, where possible, from RF currents. This is not as easy to do for current crossing the seal as for current in the direction of the seal. An ideal location for this is at the iris protruding from the short wall of the waveguide. RF current is concentrated on the iris, running along the iris at the midplane of the guide. Extending the metallization into the heavy niobium portion of the iris transfers this current to the niobium and away from the braze metallization. MAFIA studies and infrared thermal imaging have shown that most of the loss occurs at the iris. 


\subsection{Interlock Trips}

Currently, interlock trips are not expected to be a serious limitation to the operation of CEBAF at energies up to $5 \mathrm{GeV}$ [4]. In going to significantly higher energies, interlock trips would be the chief constraint on the energy reach of the machine using the current coupler configuration. The simplest approach is to remove the window from a position of exposure to the electron and x-ray flux from the cavity. Consequently, prototypes were built incorporating the window into the end of a waveguide extension which joins the cavity to the wall of the $2 \mathrm{~K}$ cryostat vessel. This extension moves the window about $15 \mathrm{~cm}$ from its original position at the cavity FPC waveguide flange. It can still view the cavity, but the solid angle is reduced. Extended windows of this style were installed on four cavities in the accelerator but interlock trips were reduced not eliminated [6].

Another extension window design, the "dogleg coupler", shields the ceramic from direct view of the cavity using an opaque undulation of the waveguide. Direct electron paths are eliminated and the undulation is deep enough to allow external lead shielding interrupting direct $x$-ray paths as well. The VSWR of the undulation is 1.06 at $1.5 \mathrm{GHz}$ and 1.013 at $2 \mathrm{GHz}$.

A prototype of this design was tested in a vertical dewar with interlock circuitry attached and the window electrically isolated such that window charging could be monitored with an electrometer. The cavity was first tested with a production window in the normal machine configuration but also electrically isolated. This window produced 7 interlock trips in 5 hours of operation with 11 watts of RF power dissipated in field emission. Window current varies from 1 to 20 nanoamperes.

A dogleg coupler was installed on the same cavity and run for 10 hours with no interlock trips. Window current was approximately $3 \times 10^{-11}$ amperes with 17 watts of RF power in field emission. Further tests are planned (Figure 4).

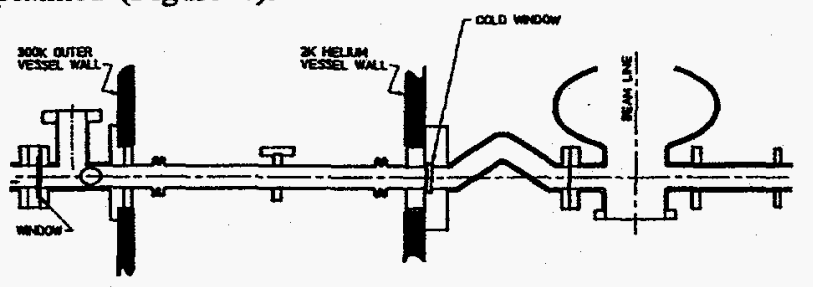

Figure 4 Dogleg Fundamental Power Coupler

The remaining issue of waveguide vacuum stability can be eliminated by using a waveguide choke joint. One side of the choke joint would be superconducting and the other side at the heat shield gas temperature. A bellows spanning the choke joint would experience a continuous thermal transition but no RF current. The thermal profile would be constant in this arrangement for all portions of the coupler below the radiation shield intercept at $50 \mathrm{~K}$.

In addition to improved pressure stability in the waveguide vacuum space, a choke joint provides the additional benefit of eliminating both RF and conduction heat loads from the waveguide into the $2 \mathrm{~K}$ bath. The design issues which must be satisfied are: 1.) adequate damping of higher order modes which are only damped through the input coupler, 2.) absence of multipacting within the choke joint, and 3.) no significant energy density within the bellows. This option is currently being explored.

\section{CONCLUSION}

Interlock trips, $2 \mathrm{~K}$ heat load, and punctures are currently limiting features of the CEBAF cold RF window design. These limitations do not significantly limit machine performance up to $5 \mathrm{GeV}$. In order to reach higher energies for the CEBAF upgrade, new window designs are required. Several modifications to the window design have been identified to accomplish this. No interlock trips have been observed in preliminary tests of a dogleg coupler design. Lower loss design options are being studied and some significant heat load reduction has been achieved.

\section{ACKNOWLEDGMENTS}

The authors would like to thank for valuable contributions: C. Reece of Jefferson Lab, E. Chojnacki of Cornell, T. Schultheis and M. Coleman of Grumman and S. Spata of Jefferson Lab.

This work was supported by the U.S. DOE under contract number DE-AC05-84ER40150.

\section{REFERENCES}

[1] Larry Phillips, Current Status of Our Understanding of Electronic Phenomena at the Cold Window. CEBAF Tech Note TN\#93-084

[2] V. Nguyen-Tuong, N. Luo, L. Phillips, C. Reece, Electronic Activity at the CEBAF Cold RF Window Induced by Cavity Operation.

[3] L. Phillips, C. Reece, T. Powers, V. Nguyen-Tuong, Some Operational Characteristics of CEBAF RF Windows at 2K, Proceedings of the 1993 Particle Accelerator.

[4] Charles Reece, Private Communications.

[5] C. E. Reece, M. Drury, M. G. Rao, V. Nguyen, Improvement of the Operational Performance of SRF cavities via in situ Helium Processing and Waveguide Processing these proceedings.

[6] J. Benesch, Private Communcations 